Revue de l'Institut des langues et cultures

d'Europe, Amérique, Afrique, Asie et Australie

$29 \mid 2017$

Les femmes en Russie : parcours, mythes et représentations

\title{
Quelques représentations de la femme dans les œuvres de Viktor Pélévine
}

НЕСКОЛЬКО ПРИМЕРОВ ИЗОБРАЖЕНИЯ ЖЕНЩИН В ПРОИЗВЕДЕНИЯХ

ВИКТОРА ПЕЛЕВИНА

Isabelle Després

\section{(2) OpenEdition}

Journals

Édition électronique

URL : http://journals.openedition.org/ilcea/4208

DOI : $10.4000 /$ ilcea.4208

ISSN : 2101-0609

Éditeur

UGA Éditions/Université Grenoble Alpes

Édition imprimée

ISBN : 978-2-37747-007-5

ISSN : $1639-6073$

Référence électronique

Isabelle Després, «Quelques représentations de la femme dans les œuvres de Viktor Pélévine », ILCEA [En ligne], 29 | 2017, mis en ligne le 30 juin 2017, consulté le 19 avril 2019. URL : http:// journals.openedition.org/ilcea/4208 ; DOI : 10.4000/ilcea.4208

Ce document a été généré automatiquement le 19 avril 2019

(C) ILCEA 


\title{
Quelques représentations de la femme dans les œuvres de Viktor Pélévine
}

\author{
НЕСКОЛЬКО ПРИМЕРОВ ИЗОБРАЖЕНИЯ ЖЕНЩИН В ПРОИЗВЕДЕНИЯХ
}

ВИКТОРА ПЕЛЕВИНА

Isabelle Després

1 Comment parler de la place de la femme dans l'univers onirique des œuvres de Viktor Pélévine, alors qu'on y trouve plutôt des êtres déshumanisés? Pourtant, le principe féminin y est bien présent, sans doute même davantage que chez d'autres auteurs des années 1990.

2 Né en 1962, V. Pélévine occupe une place incontournable dans la littérature russe des années post-soviétiques, particulièrement dans les années 1990, où ses œuvres ont suscité des réactions fortes et contrastées, car il s'agissait d'un type d'écriture nouveau, lié à la déconstruction des mythes et au jeu postmoderniste. Ainsi dans ses premiers textes, V. Pélévine revisite, sur le mode parodique et satirique, le mythe soviétique dans lequel la femme se bat aux côtés de l'homme (c'est le cas d'Anna, dans La mitrailleuse d'argile1), mais aussi le mythe «capitaliste » de la femme en tant qu'objet sexuel, faible et sotte (telle que Marina dans La vie des insectes ${ }^{2}$, ou Prosto Maria dans La mitrailleuse d'argile, ou encore Nika, dans la nouvelle du même nom).

3 Un des procédés caractéristiques du style de V. Pélévine est de gommer les frontières entre l'animal et l'humain. Le motif de la femme-animale est récurrent dans les textes de V. Pélévine (La vie des insectes, " $\mathrm{Nika}^{3}$ », Le livre sacré du loup-garou ${ }^{4}$ ). Loin de dévaloriser le personnage féminin, ce procédé permet un regard décalé et relativement original.

4 Dans les œuvres de V. Pélévine, la femme apparaît, le plus souvent, dans un couple, formant un contraste avec l'homme. Or dans ce couple, c'est la femme qui domine, ce qui peut être considéré comme une déconstruction du modèle existant, en particulier en Russie. Mais il existe d'autres modèles de civilisation, et V. Pélévine s'intéresse tout particulièrement au bouddhisme et aux mythologies orientales, où les divinités féminines 
ont parfois davantage de pouvoir que les dieux masculins. C'est le cas de la déesse Ishtar ( Homo Zapiens ${ }^{5}$, Empire $\left.V^{6}\right)$. Toutefois, même douée d'une force créatrice supérieure à celle de l'homme, la femme ne peut rien, dans ce monde du simulacre et de l'illusion, comme l'illustrent la déchéance d'Ishtar, mais aussi le cas de Vera Pavlovna?

\section{Déconstruction des mythes}

\section{La femme égale de l'homme}

5 Dans La mitrailleuse d'argile, le héros, Piotr Poustota, n'est autre que le célèbre compagnon de Vassili Tchapaïev, figure légendaire du livre de D. Fourmanov8. Dans ce roman, Anka, la figure féminine du trio (ou quatuor si on ajoute Fourmanov lui-même, le commissaire politique, qui dans le roman de V. Pélévine est évincé), peut être considérée comme emblématique de la représentation de la femme soviétique idéale: enthousiaste, active, courageuse, d'origine modeste (simple travailleuse), naïve, elle remplit aussi bien qu'un homme sa fonction à la guerre (serveuse de canon), elle partage la vie des hommes dans un esprit de franche camaraderie.

6 C'est un tout autre personnage que décrit V. Pélévine, par les yeux de Poustota, dont une des hypostases est, il faut le préciser, celle d'un poète de l'Âge d'argent.

Ses cheveux étaient très courts - il était même difficile d'appeler cela une coiffure. Un collier de grandes perles tombait sur sa poitrine à peine formée, moulée dans du velours sombre. Ses épaules étaient larges et fortes, et ses hanches un peu étroites. Ses yeux légèrement bridés ajoutaient à son charme.

Indiscutablement elle aurait pu être un étalon de beauté, mais il était difficile de qualifier cette beauté de féminine. Même dans mes moments de fantaisie la plus débridée, je me serais senti incapable de transporter ces yeux, ce visage et ces épaules dans l'obscurité chaude d'une alcôve. Oh, non! Elle convenait encore moins à ces granges à foin décrites par Bounine, où l'on attrapait la blennorragie. En revanche, on pouvait facilement l'imaginer, par exemple, sur la glace d'une patinoire. Sa beauté contenait quelque chose de dégrisant, de simple et d'un peu triste. Je ne veux pas parler de cette pudeur décorative et lascive qui indisposait tout le monde à Saint-pétersbourg, avant la guerre. Non, c'était une perfection naturelle et véritable, consciente d'elle-même, et face à laquelle la concupiscence devenait aussi ennuyeuse et banale que le patriotisme d'un flic.

Elle me regarda, se tourna vers Tchapaïev et les perles brillèrent sur son cou nu.

- C'est bien notre nouveau commissaire? demanda-t-elle.

Elle avait une voix un peu sourde, mais agréable. Tchapaïev hocha la tête.

- Piotr, Anna, nous présenta-t-il. (p. 97-989)

7 Comme le remarque le critique A. Pavlov (n. d.), son portrait est plus proche de celui de la Belle Dame que de la bonne camarade. Elle est d'une beauté fine, racée, « émancipée ». Il y a une dimension aristocratique dans le personnage. C'est une beauté calme et inaccessible, peut-être une tentatrice démoniaque (une Succube). Plus loin on la voit délicatement remuer son café avec une petite cuillère. Au chapitre 5 , elle lit un livre de Hamsun (p. 140). Elle boit du champagne, elle utilise des porte-cigarettes (p. 152). Il est d'ailleurs significatif que V. Pélévine, à propos de la beauté d'Anna, cite à plusieurs reprises l'écrivain Ivan Bounine, dont la prose est symbole de raffinement.

Une scène dans le restaurant rappelle les ambiances lourdes et décadentes de certains poèmes de A. Blok (le cycle « Carmen »), bien loin du Tchapaïev de Fourmanov (alors que dans le roman de V. Pélévine le personnage de Tchapaïev, lui, est un rustre, un paysan, 
fort éloigné du monde aristocratique pétersbourgeois). Dans La mitrailleuse d'argile, on retrouve le motif du triangle amoureux si répandu à l'époque de A. Blok, puisque Poustota a un concurrent pour la conquête du cœur d'Anna, qui est Kotovski.

Il y a donc une inversion presque totale du mythe soviétique de Anka. Ici elle est une femme puissante et intelligente, elle maîtrise le langage, elle a des conversations philosophiques avec Piotr Poustota, qui l'interroge à chaque fois qu'il se réveille d'un nouveau rêve. C'est elle qui détient le fil de la vie de Poustota, elle qui peut combler ses trous de mémoire, lui raconter l'enchaînement des événements. Elle est vraisemblablement l'infirmière de l'hôpital psychiatrique où il est soigné, mais c'est elle aussi qui manœuvre la mitrailleuse d'argile, dont la disparition à la fin du roman marque la fin du monde, ou plutôt la fin de l'illusion, la fin du délire. On retrouvera cette toute puissance de certaines femmes dans d'autres œuvres de V. Pélévine.

\section{La femme-objet}

10 Il y a un autre personnage féminin dans le roman La mitrailleuse d'argile, c'est celui de Maria. Il est inspiré, ou plutôt détourné, de celui du feuilleton mexicain télévisuel Simplement Maria, qui connaissait un immense succès en Russie au début des années 1990. C'est l'histoire d'une simple paysanne naïve qui vient à la ville pour y trouver un avenir meilleur, et tombe amoureuse d'un jeune homme riche, Juan Carlos, dont elle est enceinte, bien que leur amour soit impossible.

11 Dans le roman de V. Pélévine, Maria est soignée à l'hôpital psychiatrique par Timour Timourovitch, c'est une «femme aux larges épaules musclées qui ressemble à un travesti» (p.57). Elle a pourtant le caractère de l'héroïne mexicaine, son sourire « mystérieux et bête » (p. 63).

12 Ici (p. 56 à 77) la typographie du texte se dédouble, à la manière postmoderniste, et les « aventures » de Prosto Maria sont données en italique. La tonalité de ce texte est mièvre, la couleur rose domine (« le vide rose de son âme», p. 59), ce qui correspond au ton des feuilletons mexicains. La thématique de la fiction télévisuelle, de l'illusion, de l'inversion du regard télévisuel (qui sera développée dans le roman Homo Zapiens) est présente. L'évocation du bien et du mal se fait au second degré, avec une visée sarcastique. À nouveau plane l'ombre de A. Blok (La Belle Dame, l'Inconnue, p. 59) et de l'Âge d'argent (la tonalité biblique, avec l'attente de l'Époux, comme dans le recueil de Blok « Le monde terrible ») pour mieux se fracasser dans une déconstruction qui provoque le rire, car l'Époux n'est finalement que l'acteur culturiste américain Schwartzenegger, symbole masculin de l'univers du film hollywoodien, avec lequel Prosto Maria effectue un envol à connotations fortement sexuelles.

Il s'avère (au chapitre 4) que Maria est « un jeune homme blond au visage efféminé » et qu'elle représente « un cas simple de dédoublement de la fausse personnalité » (p. 107). Le mythe de l'héroïne sensible et au cœur compatissant est détourné, par V. Pélévine, en un homosexuel travesti, obsédé par la force brute et métallique, et par les symboles phalliques. Les frontières des genres sont effacées. Ni la femme comme égale de l'homme, ni la femme naïve et soumise ne sont épargnées par la déconstruction postmoderniste de V. Pélévine. 


\section{La femme animale}

14 Mais c'est tout simplement la nature humaine de la femme (comme, d'ailleurs, celle de l'homme) qui devient illusoire et irréelle dans les romans de V. Pélévine. Les personnages ne sont pas vraiment humains, ce sont au mieux des chimères à demi-humaines, des lycanthropes (Le livre sacré du loup-garou), mais parfois de misérables insectes (La vie des insectes), des poulets («L'Ermite et Six-Doigts»), des vampires (Empire V)... On n'a plus seulement affaire à un simple dédoublement de la personnalité, c'est un dédoublement de la nature, humaine et animale.

Dans La vie des insectes, une des premières œuvres de V. Pélévine, l'un des personnages principaux, Marina, est une jeune fille-fourmi, dont la vie est décrite de deux points de vue.

Marina entendit un bruissement d'ailes au-dessus d'elle. Elle leva la tête pour assister à l'atterrissage de deux autres fourmis pondeuses qui répétaient les manœuvres qu'elle venait de faire. Un sac comme celui de Marina pendait sur l'épaule de chacune d'entre elles et elles étaient également habillées d'une jupe en toile de jean, d'une blouse de coopérative et d'escarpins rouges à talons aiguilles ${ }^{10}$. (p.37)

16 C'est cette incertitude sur la focalisation qui fait l'intérêt de ce cycle, comme des autres romans de Pélévine de cette époque, tels que La flèche jaune ${ }^{11}$, Omon $R a^{12}$. On pourrait parler d'anthropomorphisme, si les personnages n'avaient des comportements si parfaitement humains. Il est clair que l'effet recherché n'est pas de s'intéresser à la vie des bêtes, mais de comparer la société humaine à une société animale, comme le font les fabulistes.

Que révèle l'histoire de Marina sur la représentation de la femme? La jeune fourmi effectue son vol nuptial, puis elle arrache ses ailes, car celles-ci l'encombrent (Marina est affublée d'un petit sac à main dans lequel se trouve une lime à ongles). Elle voit le monde avec joie et optimisme.

Le monde autour d'elle était beau. Il lui était particulièrement difficile de dire en quoi précisément consistait cette beauté. Pris individuellement, les objets qui l'entouraient, arbres, bancs, nuages, passants, n'étaient pas particulièrement esthétiques, mais l'ensemble produisait une claire promesse de bonheur, comme une parole d'honneur donnée par la vie. (p. 38)

18 Mais le bonheur n'est qu'un film vidéo, projeté par un téléviseur-aquarium dans un bar, qui lui promet une vie romantique, à la manière de la série télévisuelle Prosto Maria: le bonheur n'est qu'une illusion, un simulacre inspiré par les médias. La jeune fourmi plonge dans une sorte de léthargie. Marina n'est pas libre de son destin, elle est prisonnière de son instinct, qui lui impose de creuser sa fourmilière (p.35). Poussée par la nécessité et par la faim, elle se rend au marché, où elle se transforme en une mégère semblable aux autres, car elle doit défendre son butin.

Une femme maigre se tenait devant elle. Elle portait une culotte tachée d'argile et une blouse déchirée. Ses yeux brillaient d'un feu sauvage et ses cheveux, pleins de terre, étaient ébouriffés. De profondes griffures marquaient ses bras et ses jambes. D'une main elle serrait contre sa poitrine une caisse en contreplaqué qui contenait des restes de nourriture, et, de l'autre, elle tenait une pelle identique à celle de Marina. À ce signe, celle-ci comprit que c'était une pondeuse, comme elle.

[...]

- Fiche le camp, salope ! cria Marina. (p. 45-46) 
femme-renarde, qui forme un couple avec un loup-garou (un lycanthrope) ${ }^{15}$. Le roman est écrit du point de vue de cette A Huli, dont l'auteur prétend avoir retrouvé une sorte de journal intime. Le nom même de ce personnage est significatif, il possède une charge de déconstruction ironique, du fait qu'il évoque une grossièreté de la langue russe. Mais il évoque aussi la mythologie chinoise, dont un motif récurrent est une femme-renarde appelée húlijīng. D’après Wikipédia, il s'agit « d'une renarde qui se métamorphose en une charmante et agréable jeune fille pour séduire les héros; la femme-renarde est décrite comme un esprit tantôt dangereux, tantôt bienfaisant ${ }^{16}$ ».

Chez V. Pélévine, cette renarde est aussi un esprit immortel, qui peut se réincarner, en particulier en une prostituée de luxe, dans le Moscou des années Eltsine. Fidèle à son style, V. Pélévine entretient l'ambiguïté en prêtant à la renarde des traits féminins (talons aiguilles, sac à main...) et animaux (un poil argenté, des cheveux d'un roux flamboyant). La renarde a l'instinct animal de la chasse (chasse aux aristocrates anglais et aux poules, qu'elle relâche, toutefois, après les avoir attrapées). Dans le portrait physique que fait d'elle-même la femme-renarde, on retrouve la référence à Lolita (p. $16^{17}$ ), et plus loin le 
héros l'appelle Ada (p.125). V. Pélévine n'a pas fini de dialoguer avec son illustre prédécesseur V. Nabokov.

Notons que ce portrait est collectif: elle décrit, à travers elle-même, une espèce de femmes, les renardes, qui n'ont que l'apparence de la féminité, car elles sont " asexuées ", elles n'ont pas d'appareil reproductif. Ces femmes ont un corps fin, svelte, musclé, « comme certains ados très sportifs » (p. 30).

Mais l'attribut principal des femmes-renardes est leur queue: " une antenne souple et duveteuse d'un roux intense. Elle peut devenir plus ou moins grande. Au repos, elle ressemble à une queue de cheval de dix à quinze centimètres, mais en activité elle peut s'étirer sur près d'un mètre » (p. 31). Ici l'auteur fait très sciemment allusion au phallus masculin.

On pourrait y voir un détournement du mythe de l'androgyne, réactualisé en Russie à l'Âge d'argent, en lien avec la philosophie de la divino-humanité de Vladimir Soloviov (le bogotchelovek), mais il ne s'agit pas tant pour la renarde de dépasser la condition sexuée, afin de dégager une énergie spirituelle créatrice, il s'agit au contraire d'exalter une forme d'hyper sexualité, de sur-sexualité.

La femme-renarde est décrite comme une forme supérieure d'être humain. La femme humaine possède certains des pouvoirs de la renarde (par exemple, le pouvoir de manipulation par la beauté), mais n'en ayant pas conscience, elle est incapable de les utiliser.

31 Finalement, le roman apparaît comme une apologie de la féminité. Le surhomme tel que l'avait rêvé le Zarathoustra de Nietzsche est une femme. Pour V. Pélévine, c'est la femmerenarde. Pourtant, cet idéal s'avère aussi trompeur.

\section{Mensonge et simulacre}

Si le postmodernisme n'est pas un anti-modernisme, mais la continuité et la fin du modernisme, alors il n'est guère étonnant que la femme, pour les postmodernistes, soit également dans la continuité de l'idéal féminin des poètes et philosophes de l'Âge d'argent. Dans Le livre sacré du loup-garou, bien des éléments fonctionnent en intertextualité avec les grands motifs de la culture russe, en particulier de l'Âge d'argent.

On a vu à quel point le portrait d'Anka dans La mitrailleuse d'argile est éloigné du personnage du film ou du roman de Fourmanov. C'est celui d'une aristocrate racée, qui fume avec un porte-cigare, et dans ses yeux il y a comme une tristesse, une sagesse, un mystère.

On a retrouvé dans le personnage de Nika ce mystère, cette inaccessibilité, ce sentiment du narrateur de ne pouvoir comprendre la femme, car elle est d'un autre monde. Selon V. Pélévine, c'est la tragédie de tous les couples, car ils ne parlent pas le même langage (Nika est quasiment muette). Même s'ils croient se rencontrer dans la relation amoureuse, il s'agit toujours d'un quiproquo.

Rama, le héros d'Empire V, ment à la jeune fille qu'il choisit pour sa « Première morsure ». Toute relation entre les êtres humains, y compris entre un homme et une femme, est vouée à l'échec, car elle repose sur le mensonge. Ainsi, dans Le livre sacré du loup garou, le client de la prostituée est trompé, la relation sexuelle n'a pas lieu, elle lui est seulement 
suggérée par la femme-renarde, sous forme d'hallucination, et en fonction de ses fantasmes.

L'androgyne, en tant qu'être ayant retrouvé sa plénitude, est un idéal pour les poètes de l'Âge d'argent (Blinova, 2012). Pour eux il s'agit de sortir des clivages traditionnels, de dépasser les oppositions binaires. Le mythe de l'androgynie s'accompagne de l'idéal «platonique» de renoncement au sexe ${ }^{18}$. Au contraire, dans le roman de V. Pélévine $L e$ livre sacré du loup-garou, tout se ramène à une forme de sexualité exacerbée, dans la langue comme dans la culture, une forme de sur-sexualité dionysiaque qui, d'ailleurs, est aussi un des traits caractéristique de l'Âge d'argent ${ }^{19}$.

V. Pélévine va plus loin dans la déconstruction des mythes de l'Âge d'argent. Pour le philosophe Vladimir Soloviov et les poètes symbolistes, il existait un principe féminin, la Sofia, qui est la sagesse, l'intelligence de la Révélation du mystère divin. C'est « l'âme du monde ", l'énergie créatrice fondamentale. Dans Le livre sacré du loup-garou, elle a pris la forme de l'énergie sexuelle, dont se nourrit A Huli pour vivre éternellement.

Dans Empire V il y a aussi une « âme » qui est la « langue » (âzyk, terme masculin en russe), et le monde post-soviétique, capitaliste, est parcouru par des fluides quasiment érotiques (le glamour et le discours). Ce n'est pas la Sagesse qui fait vivre le monde postmoderne, mais le sexe, le glamour et le discours (on pourrait ajouter le pétrole qui jaillit de la terre, dans l'épisode où le loup-garou fait sa prière au Veau d'or...).

\section{La Déesse créatrice de l'univers}

On l'a vu avec le personnage de A Huli, la femme chez Pélévine a souvent des pouvoirs surnaturels. Elle est immortelle, elle peut se métamorphoser, manipuler les hommes. Dans la nouvelle "Nika ", la femme est un chat, ce qui n'est pas sans rappeler la déesse à tête de chat de la mythologie égyptienne. Mais c'est dans Homo Zapiens, puis dans Empire $V$ que Dieu devient une femme, Ishtar.

Homo Zapiens est construit sur le motif de Babylone et de la tour de Babel, motif récurrent de la poétique des écrivains russes postmodernistes (Genis, 1997), qui symbolise l'écroulement d'un monde hiérarchisé et orienté vers le haut, vers le ciel, l'Absolu. Le mythe exprime aussi, avec la naissance des diverses langues, la perte d'un langage commun à l'humanité, et donc le début de l'incommunicabilité fondamentale entre les humains.

41 L'intérêt de V. Pélévine pour la mythologie orientale était déjà évident dans La mitrailleuse d'argile, mais dans Homo Zapiens il s'agit plus précisément de la mythologie babylonienne. L'ascension sociale de Vavilen Tatarski s'accompagne d'une ascension pseudo-spirituelle, favorisée par sa consommation de stupéfiants. Il parvient ainsi en haut de la pyramide sociale, et découvre parallèlement que le monde est au service de la déesse Ishtar, qui a su séparer son moi mortel (le chien à cinq pattes qui dort quelque part dans le Nord, s'il se réveille les catastrophes commencent ${ }^{20}$ ) et son moi immortel (tout l'or du monde). Tatarski devient le nouvel « époux » d'Ishtar. Après ce mariage sacré, il devient le roi de Babylone, et, surtout, il prend la tête de la Compagnie, à la place d'Azadovski. Son attitude vis-à-vis de la secrétaire Alla change du tout au tout. Il devient abusivement autoritaire.

Remarquons au passage que la secrétaire Alla est comparée à une méduse pécheresse, à cause de ses longs cheveux clairs détachés. Nous retrouvons là une représentation de la femme animale, dont nous avons déjà parlé. Elle apparaît ici comme l'initiatrice de 
Tatarski aux mystères du monde (elle lui fait découvrir la collection de tableaux espagnols), et ensuite comme sa servante (sa secrétaire personnelle).

Dans la représentation postmoderniste de Pélévine, l'univers se ramène à l'imaginaire subconscient du héros mâle, dont il n'est qu'une hallucination. Puisque le monde n'est que sa «création » fantasmée, Ishtar la créatrice se confond avec le désir de l'homme, il est logique qu'elle soit une divinité féminine, une Déesse et non un Dieu. Toutefois, dans Homo Zapiens, la Déesse n'est pas décrite, elle n'est pas objectivée, elle n'a pas d'existence précise, ni de représentation, dans l'imagination du héros. Elle n'existe que grâce à l'intertexte des légendes babyloniennes.

Dans Empire V également, la déesse Ishtar siège au sommet de la pyramide (ou, peut-être, au fond, car cette pyramide, tout compte fait, et comme dans Homo Zapiens, est inversée, de sorte qu'Ishtar se trouve dans la chambre mortuaire au plus profond de la pyramide). Elle s'incarne dans une femme-vampire.

n'a cependant pas de réel pouvoir. Ishtar a été bannie pour un crime inconnu, et la Terre est sa prison. Les vampires avaient pour tâche d'expliquer à la déesse pourquoi elle avait créé le monde, mais ils n'ont pas réussi! On se trouve donc dans un monde absurde et vide, conforme à la représentation postmoderniste. Lorsque le héros découvre la Déesse, après avoir parcouru une enfilade de salles de musée, retraçant toute l'histoire de l'humanité, et qu'il pénètre enfin dans la salle du trône, elle est à moitié saoule. Elle a l'aspect d'un champignon géant, elle n'est qu'une simple tête posée sur un cou.

Du centre de la niche, me regardait, avec un sourire, un visage féminin, de ceux dont on dit qu'il leur reste des traces de leur beauté passée. La tête avait près d'une cinquantaine d'années, à première vue, mais sans doute plus, car même moi, qui ne suis pas très au fait de ces choses, je remarquai les traces des multiples interventions chirurgicales et cosmétiques de rajeunissement. Seule la bouche souriait, les yeux encerclés d'une peau figée, exprimaient le doute et l'angoisse ${ }^{21}$.

Ishtar sera bientôt engloutie par la vieillesse et l'impotence. Déjà elle n'a plus la force de se verser du cognac toute seule. C'est une Déesse déchue, monstrueuse, abjecte.

\section{La mère décrépite}

Or, lors de leur rencontre, Ishtar rappelle au héros son enfance « malheureuse ». Au début du roman, on apprend que le héros vient de rompre avec sa mère et, ainsi, de quitter le monde de l'enfance. Un chapitre entier, le second, est consacré à ce passé radieux qui, pour le héros, est à la fois celui de l'enfance et de l'URSS. La mère du héros y apparaît comme sûre d'elle, autoritaire, toute puissante, écrasant psychologiquement son fils (ils vivent sans le père, qui les a quittés dès la naissance du fils).

Elle était sûre que je prenais de la drogue et se croyait capable de savoir quand j'étais drogué ou non [...]. Je ne la contredisais pas, comprenant que cela aurait été une preuve supplémentaire qu'elle avait raison ("Que tu es agressif quand tu te drogues, c'est effrayant !»).

En outre ma mère avait une force d'hypnose hors du commun. Il lui suffisait de dire : «Tu mélanges les mots! », et je commençais en effet à mélanger les mots [... $]^{22}$

En la quittant, le héros la désacralise, et se débarrasse du complexe d'‘Edipe. C'est une des grilles de lecture du roman Empire $V$. Il apparaît ainsi que la déesse Ishtar incarne, non pas le principe féminin de la Belle Dame, de l'amante idéale, mais plutôt celui de la mère, celle qui meurt pour redonner la vie, dont la décrépitude est nécessaire pour que l'enfant 
se métamorphose. C'est une mère-vampire, une mère chauve-souris, bien qu'elle ait enfanté le monde. Dans Empire $V$, le héros évite de justesse l'inceste, mais finalement la Déesse s'est choisi un autre vampire pour en faire sa victime sacrificielle.

Au delà du simulacre psychanalytique, on retrouve ici un vague écho de la figure de la Terre nourricière, de la Terre-mère-humide, qui est une constante de la tradition folklorique, associée à la Russie. Le corps de la Russie est un corps féminin, qui connaît des « jours critiques », c'est-à-dire des périodes de crise, où le sang coule ${ }^{23}$.

\section{La créatrice impuissante}

Pour finir, arrêtons-nous sur la déconstruction du mythe de l'héroïne positive, telle que l'incarne Vera Pavlovna, le personnage féminin de Que faire?, roman culte des radicaux et révolutionnaires russes du XIX siècle. Dans la nouvelle de V. Pélévine, intitulée "Le neuvième songe de Vera Pavlovna ${ }^{24}$ ", le personnage central est une femme modeste et discrète, qui travaille comme femme de ménage dans les toilettes publiques à Moscou. Malgré sa situation sociale, sa vie intérieure est aussi riche que celle de son prototype du roman de Nikolaï Tchernychevski. Elle se pose des questions philosophiques sur le sens de l'existence, et elle a une âme d'artiste et elle aime la musique de Bach. Par la force de son imagination et de sa volonté, elle parvient à métamorphoser son petit monde étriqué des toilettes publiques en un monde agrandi, augmenté, relativement confortable.

51 En réalité, à l'extérieur, l'époque change, mais Vera n'en voit que le reflet dans les toilettes. Dans un premier temps les toilettes sont privatisées en une coopérative, puis elles sont rachetées par un riche individu pour en faire un grand magasin, et le petit monde magique de Vera est voué à la disparition. Les toilettes sont une métaphore du pays tout entier, qui est passé de l'URSS à la Russie de Eltsine.

- J'ai peur que tout ne soit pas aussi simple. Bien sûr, d'un côté en effet nous créons tout ce qui nous entoure, mais de l'autre nous sommes nous-mêmes le simple reflet de ce qui nous entoure. C'est pourquoi tout destin individuel, quel que soit le pays, est la répétition métaphorique de ce qui arrive au pays, et ce qui arrive au pays est constitué des milliers de vies individuelles.

[...]

Et là Vera comprit que pendant qu'elle dirigeait le monde, elle s'était fait rattraper par sa vieillesse, et devant elle il n'y avait plus que sa mort ${ }^{25}$.

Alors Vera fait un rêve, où Moscou, comme une Atlantide, disparaît sous les eaux d'une gigantesque inondation due à une rupture de canalisation, qui emporte tout. La nouvelle se termine sur un constat d'impuissance et de renoncement, dans une répétition parodique, sarcastique, et sans aucun doute métaphorique, du roman de Tchernychevski.

- Que faire ? répéta Vera, - comment ça, que faire? Et soudain ce fut comme un grand coup de vent. Ce n'était pas du vent, mais cela y faisait penser, car Vera sentit qu'elle était emportée comme une feuille arrachée par le vent.

- Que faire ? répéta Vera, comme par inertie. Et soudain elle comprit tout ${ }^{26}$.

Comme en témoignent les dernières lignes de la nouvelle, empruntées directement au chapitre XXVI de Que faire?, Vera Pavlovna se retrouve emprisonnée dans la prose du roman de Tchernychevski, ce qui est le comble de son impuissance et de son échec à réinventer le monde. 


\section{Conclusion} qu'un jeu postmoderniste de déconstruction des mythes. Fidèle à son écriture, et grâce à un procédé de focalisation multiple, V. Pélévine s'amuse à remettre en question les frontières entre les genres, entre les espèces, entre les espaces-temps, il nie la possibilité d'une perception objective de la réalité, d'une communication réelle et sincère entre les individus.

Femme soviétique, femme-objet, femme animale, femme manipulatrice, femme toute puissante, femme créatrice, femme-mère, toutes les hypostases traditionnelles de la femme sont revisitées et déconstruites sur le mode de l'ironie et du sarcasme, par les allusions intertextuelles, la parodie des mythes littéraires et culturels, le deuxième degré. V. Pélévine n'épargne aucun des textes culturels fondateurs, pas davantage celui de l'Âge d'argent, ceux de I. Bounine ou V. Nabokov, que le texte "soviétique» de Fourmanov. Il désacralise, certes, la culture russe du XIX et du $\mathrm{Xx}^{\mathrm{e}}$ siècle, mais l'objet de sa critique est, avant tout, la Russie post-soviétique, celle du pouvoir politique corrompu, allié au règne de l'argent et du pétrole, celle de la consommation absurde, de la confusion, de l'illusion et du simulacre.

57 Aucun modèle féminin n'échappe à cette ironie corrosive, sauf peut-être celui de la femme-renarde du Livre sacré du loup-garou, seul exemple où la narration est menée d'un point de vue féminin, et où le portrait psychologique de l'héroïne acquiert un peu d'épaisseur (en particulier, son sentiment amoureux n'est pas entièrement illusoire). Toutefois, même si les créatures féminines de V. Pélévine ne résistent pas à la déconstruction, et deviennent des insectes, des chauves-souris (Ishtar), des chattes (Nika), ou des clones de personnages littéraires (Vera Pavlovna), l'auteur garde pour elles, du moins dans les œuvres dont il a été question dans cet article ${ }^{27}$, une certaine tendresse : elles sont moins corrompues, moins cyniques, souvent plus intelligentes et sensibles, que leurs partenaires masculins. Pour l'auteur, le mystère de la femme semble rester entier, ainsi que l'attirance qu'elle suscite. 


\section{BIBLIOGRAPHIE}

BuInova Olga (2012), Le mythe de l'androgyne dans l'œuvre poétique de Zinaida Gippius (thèse de doctorat), Strasbourg.

GENIS Alexandr (1997), «Vavilonskaâ bašnâ, iskusstvo nastoâŝego vremeni », Nezavisimaya Gazeta.

PAvlov A. (n. d.), «Arxetipy massovogo soznaniâ v romane V. O. Pelevina "Čapaev i Pustota" ", [3, 135], <www.mineralov.su/pavl.htm>.

Pelevin Viktor (1996a), Čapaev i Pustota. Traduction française de Galia Ackerman et Pierre Lorrain, La mitrailleuse d'argile, Paris : Seuil, 1997.

Pelevin Viktor (1996b), Sočineniâ $v$ dvux tomax, Moscou : Terra.

PeLEVIN Viktor (1999), Generation P. Traduction française de Galia Ackerman et Pierre Lorrain, Homo Zapiens, Paris : Seuil, 2001.

PeLEVIN Viktor (2004), Svâsennaâ kniga oborotnâ. Traduction française de Galia Ackerman et Pierre Lorrain, Le Livre sacré du loup-garou, Paris : Denoël, 2009.

PELEVIN Viktor (2006), Empire V, povest' o nastoâsem sverxčeloveke, Moscou : Eksmo.

RONDEL Aude (2011), Le loup-garou dans la littérature contemporaine. De l'imaginaire fictionnel aux mises en scènes sociales, Paris : Publibook.

SVETLAKova Mariâ (2015), «Ženskie obrazy v tvorčestve Pelevina », <http:// msvetlakova.livejournal.com/9838.html>.

\section{NOTES}

1. En russe, Čapaev i Pustota, paru en 1996. Traduction française de G. Ackerman et P. Lorrain, La mitrailleuse d'argile, Seuil, 1997.

2. En russe, "Žizn' nasekomyx ", Znamâ, no 4, 1993. Traduction française de G. Ackerman et P. Lorrain, La vie des insectes, Seuil, 1995.

3. La nouvelle « Nika » a été publiée dans Pelevin (1996b, t. 2, p. 289-301). Non traduit.

4. En russe Svâŝennaâ kniga oborotnâ, paru en 2004. Traduction française de G. Ackerman et

P. Lorrain, Le Livre sacré du loup-garou, Denoël, 2009.

5. En russe Generation P., paru en 1999. Traduction française de G. Ackerman et P. Lorrain, Homo Zapiens, Seuil, 2001.

6. Empire V, povest' o nastô̂ŝem sverxčeloveke, Eksmo, 2006. Non traduit en français.

7. «Devâtyj son Very Pavlovny », écrit en 1991, publié dans Pelevin (1996b, t. 1, p. 297-316). Le récit est un pastiche des songes d'avenir radieux de Vera Pavlovna, dans le roman de N. Tchernychevski Que faire?

8. C'est le film des frères Vassilev (1935) qui a rendu ce roman aussi célèbre.

9. V. Pelevin, La mitrailleuse d'argile, trad. G. Ackerman et P. Lorrain, Seuil, 1997. Ici et plus loin les numéros des pages renvoient à cette édition.

10. Ici et plus loin, le numéro de page renvoie à l'édition traduite de G. Ackerman et P. Lorrain (1997). 
11. En russe, Želtaâ strela, paru dans la revue Novy Mir en 1993. Traduction française de G. Ackerman et P. Lorrain, La flèche jaune, Denoël, 2006.

12. Omon $R a$, 1992. Traduction française de G. Ackerman et P. Lorrain, Omon Ra, Mille et une nuits, 1998.

13. On aura noté le jeu de mots sur « pelle », qui se dit « sovok », mot méprisant des années 1990 pour désigner le « soviétique ».

14. «<...> ГОТОВЫЙ ОЦЕНИТЬ ЕЕ СМУГЛУЮ ЮЖНУЮ ПРЕЛЕСТЬ И СМЯГЧИТЬ ЕЙ ТЯЖЕСТЬ СУЩЕСТВОВАНИЯ ВДАЛИ ОТ ДРЕВНЕЙ РОДИНЫ, В ГОЛОДНОЙ СЕВЕРНОЙ СТРАНЕ, ГДЕ ОНА ПО НЕДОРАЗУМЕНИЮ РОДИЛАСЬ. КОГДА ОНА ПРЯТАЛА ГОЛОВУ У МЕНЯ НА ГРУДИ, Я МЕДЛЕННО ПРОВОДИЛ ПАЛЬЦАМИ ПО ЕЕ ШЕЕ <..>. » Traduit par l'auteur de l'article.

15. Voir aussi Rondel (2011).

L'auteur compare et analyse trois auteurs contemporains de langues diverses (anglais, russe, français), parmi lesquels figure Pélévine.

16. <https://fr.wikipedia.org/wiki/Femme-renarde>.

17. Ici et plus loin les numéros des pages renvoient à la traduction française parue chez Denoël en 2009.

18. Voir le mythe de l'androgyne dans Le Banquet de Platon.

19. Voir en particulier Viatcheslav Ivanov et ses travaux sur la religion dionysiaque : <http:// rvb.ru/ivanov/>.

20. Ce chien peut être vu comme une des variantes du loup-garou, motif récurrent de la prose de V. Pélévine, depuis ses premiers récits jusqu'au Livre sacré du loup-garou.

21. « ИЗ ЦЕНТРА НИШИ НА МЕНЯ С УЛЫБКОЙ СМОТРЕЛО ЖЕНСКОЕ ЛИЦО - КАК ЭТО ГОВОРЯТ, СО СЛЕДАМИ КОГДА-ТО БЫВШЕЙ КРАСОТЫ. ГОЛОВЕ НА ВИД БЫЛО ОКОЛО ПЯТИДЕСЯТИ ЛЕТ, А НА САМОМ ДЕЛЕ НАВЕРНЯКА БОЛЬШЕ, ПОТОМУ ЧТО ДАЖЕ МНЕ, НЕ ОСОБО НАБЛЮДАТЕЛЬНОМУ В ТАКИХ ВЕЩАХ, БЫЛИ ЗАМЕТНЫ СЛЕДЫ МНОГОЧИСЛЕННЫХ КОСМЕТИЧЕСКИХ ПРОЦЕДУР И ОМОЛАЖИВАЮЩИХ УКОЛОВ. УЛЫБАЛСЯ ОДИН РОТ, А ОКРУЖЕННЫЕ НЕПОДВИЖНОЙ КОЖЕЙ ГЛАЗА ГЛЯДЕЛИ С СОМНЕНИЕМ И ТРЕВОГОЙ. »(Pelevin, 2006, p. 245) Traduit par l'auteur de l'article.

22. « ОНА БЫЛА УВЕРЕНА, ЧТО Я ПРИНИМАЮ НАРКОТИКИ, И СЧИТАЛА СЕБЯ СПОСОБНОЙ ОПРЕДЕЛИТЬ, КОГДА Я ПОД КАЙФОМ, А КОГДА НЕТ. <... Я И НЕ СПОРИЛ, ПОНИМАЯ, ЧТО ЭТО БУДЕТ ЛИШНИМ ДОКАЗАТЕЛЬСТВОМ ЕЕ ПРАВОТЫ (“КАКОЙ ТЫ АГРЕССИВНЫЙ СТАНОВИШЬСЯ ПОД НАРКОТИКАМИ, УЖАС ПРОСТО!").

КРОМЕ ТОГО, МАТЬ ОБЛАДАЛА ИЗРЯДНОЙ ГИПНОТИЧЕСКОЙ СИЛОЙ: СТОИЛО ЕЙ, НАПРИМЕР, СКАЗАТЬ: “ДА

У ТЕБЯ ЖЕ СЛОВА ПРЫГАЮТ!" - КАК У МЕНЯ ДЕЙСТВИТЕЛЬНО НАЧИНАЛИ ПРЫГАТЬ СЛОВА <...>.» (Pelevin, 2006, p. 23-24) Traduction de l'auteur de l'article.

23. Dans un des chapitres de Homo Zapiens intitulé «Les jours critiques", à propos de la crise politique de 1993, V. Pélévine a recours à la métaphore des règles (l'esprit perverti du héros en fait immédiatement une publicité pour les tampons Tampax: «- НОРМАЛЬНО, - СКАЗАЛ ТАТАРСКИЙ И МЕЧТАТЕЛЬНО ЦОКНУЛ ЯЗЫКОМ. — СЛОГАН ПРОСИТСЯ: “ТАМПАКС ultra safe: КРАСНЫЕ НЕ ПРОЙДУТ!" »)

24. Voir note 7.

25. « - БОЮСЬ, ВСЕ НЕ ТАК ПРОСТО. КОНЕЧНО, С ОДНОЙ СТОРОНЫ МЫ ДЕЙСТВИТЕЛЬНО СОЗДАЕМ ВСЕ ВОКРУГ, НО С ДРУГОЙ - МЫ САМИ ПРОСТО ОТРАЖЕНИЯ ТОГО, ЧТО НАС ОКРУЖАЕТ. ПОЭТОМУ ЛЮБАЯ ИНДИВИДУАЛЬНАЯ СУДЬБА В ЛЮБОЙ СТРАНЕ - ЭТО МЕТАФОРИЧЕСКОЕ ПОВТОРЕНИЕ ТОГО, ЧТО С ПРОИСХОДИТ СО СТРАНОЙ, А ТО, ЧТО ПРОИСХОДИТ СО СТРАНОЙ, СКЛАДЫВАЕТСЯ ИЗ ТЫСЯЧ ОТДЕЛЬНЫХ ЖИЗНЕЙ.

$<\ldots>$

И ТУТ ВЕРА ПОНЯЛА, ЧТО ПОКА ОНА УПРАВЛЯЛА МИРОМ, К НЕЙ ПРИШЛА СТАРОСТЬ, И ВПЕРЕДИ ТЕПЕРЬ тольКо СмЕРть. » (Pelevin, 1996b, t. 1, p. 307) Traduction de l'auteur de l'article. 
26. « - ЧТО ДЕЛАТЬ? - ПЕРЕСПРОСИЛА ВЕРА, - КАК ЧТО ДЕЛАТЬ? И ВДРУГ ВОКРУГ СЛОВНО ПОДУЛ ВЕТЕР - ЭТО НЕ БЫЛО ВЕТРОМ, НО НАПОМИНАЛО ЕГО, ПОТОМУ ЧТО ВЕРА ПОЧУВСТВОВАЛА, ЧТО ЕЕ КУДАТО НЕСЕТ, КАК ПОДХВАЧЕННЫЙ ВЕТРОМ ЛИСТ.

- ЧТО ДЕЛАТЬ? - ПО ИНЕРЦИИ ПОВТОРИЛА ВЕРА И ВДРУГ ВСЕ ПОНЯЛА.» (Pelevin, 1996b, t. 1, p. 315) Traduction de l'auteur de l'article.

27. Nous n'avons pas pris en compte dans cet article les œuvres plus récentes de Pélévine, telles que S.N.U.F.F. ou Une eau d'ananas, où la femme, d'après un article de la blogueuse Maria Svetlakova (2015), est réduite à un être passif conforme aux fantasmes masculins les plus dégradants.

\section{RÉSUMÉS}

L'article examine quelques représentations de la femme dans les œuvres de V. Pélévine, écrivain russe contemporain à grand succès, représentatif du postmodernisme. On s'intéresse d'abord aux personnages féminins de La Mitrailleuse d'argile, où, dans un dialogue intertextuel avec le mythe de la Belle Dame de l'Âge d'argent, est déconstruit le mythe soviétique de la femme égale de l'homme, ainsi que le stéréotype de la femme-objet de la société capitaliste. Puis sont étudiés les personnages femmes animales, telles que femme-renarde du Livre sacré du loup-garou. On montre ensuite la déesse Ishtar, une femme décrépite occupant la place de Dieu, dans Homo Zapiens et dans Empire $V$, pour finir sur la rêveuse, la créatrice impuissante de la nouvelle « Le neuvième songe de Vera Pavlovna ». L'article conclut que dans ces textes, sous le vernis de l'ironie, du simulacre et de la déconstruction, reste perceptible une certaine tendresse pour les personnages féminins.

В СТАТЬЕ РАССМАТРИВАЮТСЯ ИЗОБРАЖЕНИЕ ЖЕНЩИНЫ В НЕКОТОРЫХ ПРОИЗВЕДЕНИЯХ РУССКОГО СОВРЕМЕННОГО ПИСАТЕЛЯ ВИКТОРА ПЕЛЕВИНА, ПРЕДСТАВИТЕЛЯ ПОСТМОДЕРНИЗМА, КОТОРЫЙ ПОЛЬЗУЕТСЯ БОЛЬШИМ ЧИТАТЕЛЬСКИМ УСПЕХОМ. РЕЧЬ ИДЁТ СНАЧАЛА О ЖЕНСКИХ ПЕРСОНАЖАХ РОМАНА ЧАПАЕВ И ПУСТОТА. ЧЕРЕЗ ИНТЕРТЕКСТУАЛЬНЫЙ ДИАЛОГ С МИФОМ СЕРЕБРЯНОГО ВЕКА О ПРЕКРАСНОЙ ДАМЕ, АВТОР СОВЕРШАЕТ ДЕКОНСТРУКЦИЮ СОВЕТСКОГО МИФА О ЖЕНЩИНЕ, РАВНОЙ МУЖЧИНЕ, А ТАКЖЕ СТЕРЕОТИПА КАПИТАЛИСТИЧЕСКОГО ОБЩЕСТВА О ЖЕНЩИНЕ-ОБЪЕКТЕ. ЗАТЕМ ИЗУЧАЮТСЯ ЖЕНЩИНЫ-ЖИВОТНЫЕ, ТАКИЕ КАК ЛИСА В СВЯЩЕННОЙ КНИГЕ ОБОРОТНЯ. УКАЗЫВАЕТСЯ НА БОГИНЮ ИШТАР, ДРЯХЛУЮ ЖЕНЩИНУ, КОТОРАЯ ЗАНИМАЕТ МЕСТО БОГА В Generation П И В Empire $V$, И НАКОНЕЦ НА МЕЧТАТЕЛЬНИЦУ, БЕСПОМОЩНУЮ СОЗДАТЕЛЬНИЦУ МИРА, ИЗ РАССКАЗА «ДЕВЯТЫЙ СОН ВЕРЫ ПАВЛОВНЫ». В ЗАКЛЮЧЕНИЕ ГОВОРИТСЯ, ЧТО НЕСМОТРЯ НА ИРОНИЧНОСТЬ, НА СТАВКУ НА СИМУЛЯКР И ДЕКОНСТРУКЦИЮ, В ЭТИХ ТЕКСТАХ ВСЁ ЖЕ ЧУВСТВУЕТСЯ НЕКОТОРАЯ НЕЖНОСТЬ ПИСАТЕЛЯ ПО ОТНОШЕНИЮ К ЖЕНСКИМ ПЕРСОНАЖАМ. 
INDEX

motsclesru РУССКАЯ СОВРЕМЕННАЯ ЛИТЕРАТУРА, ПОСТМОДЕРНИЗМ, ОБРАЗ ЖЕНЩИНЫ, ДЕКОНСТРУКЦИЯ, ПЕЛЕВИН

Mots-clés : littérature russe contemporaine, postmodernisme, représentation de la femme, déconstruction, Pélévine

\section{AUTEUR}

\section{ISABELLE DESPRÉS}

Université Grenoble Alpes, CESC/ILCEA4 